\title{
La política de la Unión Europea en Oriente Medio, historia y actualidad
}

\section{European Union Policy in the Middle East, History and Current Affairs}

\author{
Najib Abu-warda ${ }^{1}$ \\ Universidad Complutense de Madrid (España)
}

Recibido: 13-10-20

Aceptado: 22-10-20

\section{Resumen}

Oriente Medio es una región de gran importancia estratégica para el resto del mundo. Dada su situación geoestratégica, Oriente Medio ha sido un cruce de caminos de importancia histórica y sigue siéndolo aún hoy. Además de su importancia estratégica, el magnetismo cultural de Oriente Medio ha atraído grandes potencias extrarregionales a la zona durante siglos. Hoy, como en el pasado, Oriente Medio desempeña un papel importante en la política exterior de la Unión Europea. Sin embargo, la Unión Europea no tiene un enfoque único y general para la conducción de sus relaciones con Oriente Medio. En cambio, tiene un conjunto de políticas entrelazadas hacia subregiones específicas, países y áreas problemáticas. Entre estas destacan las relaciones euromediterráneas; el conflicto palestino-israelí; el diálogo UE-CCG; la cuestión nuclear iraní; las relaciones con Turquía; la guerra en Siria, entre otras. Este estudio se centra en la política de la Unión Europea en estos espacios y cuestiones que, hoy, siguen siendo temas de máxima actualidad regional e internacional.

Palabras-clave: Unión Europea, Oriente Medio, política exterior, historia, actualidad.

\footnotetext{
${ }^{1}$ (abuwardanajib@gmail.com). Profesor de Relaciones Internacionales. Entre sus obras destacan: Estados Unidos y el proceso de paz palestino-israeli: "El Pacto del Siglo" (en AAVV, "El sistema internacional del siglo XXI", Valencia, Tirant lo Blanch, 2020); La Liga de los Estados Árabes ante los acontecimientos regionales (en AAVV, "La Primavera Árabe y sus perspectivas regionales e internacionales", Madrid, Diwan Mayrit, 2018); : Los medios de comunicación árabes, estructura y características, Revista ZER, 2000; Las relaciones internacionales en la concepción islámica, Revista de Estudios Internacionales, UCM, 1999, 1.
} 


\begin{abstract}
The Middle East is a region of great strategic importance to the rest of the world. Given its geostrategic situation, the Middle East has been a crossroads of historical importance and remains so to this day. In addition to its strategic importance, the cultural magnetism of the Middle East has attracted great extraregional powers to the area for centuries. Today, as in the past, the Middle East plays an important role in eu foreign policy. However, the EU does not have a single and comprehensive approach to conducting its relations with the Middle East. Instead, it has a set of policies intertwined towards specific subregions, countries and problem areas. These include Euro-Mediterranean relations; The Israeli-Palestinian conflict; The EU-GCC dialogue; The Iranian nuclear issue; Relations with Turkey; The war in Syria, among others. This study focuses on EU policy in these areas and issues, which, to date, remain the most topical regional and international policy issues.
\end{abstract}

Key-words: European Union, Middle East, Foreign Policy, History, Current Affairs.

\title{
Introducción
}

La Unión Europea, como organización internacional supranacional, tiene su base constitucional en los Tratados internacionales concluidos por los Estados miembros. La política exterior y de seguridad común (PESC), instaurada en el Tratado de Maastricht, tiene entre otros objetivos el fomento de la cooperación internacional con terceros Estados, el desarrollo, la consolidación de la democracia y el Estado de Derecho, así como el respeto de los Derechos Humanos y de las Libertades Fundamentales. Para ello, la Unión Europea mantiene asociaciones y diálogos políticos con los principales actores internacionales. En 1997, el Tratado de Ámsterdam estableció un proceso de decisión más eficaz que incorporaba la abstención constructiva y las votaciones por mayoría cualificada. En 1999, el Consejo Europeo creó la función de alto representante para la PESC. En el Tratado de Niza de 2003 se introdujeron otros cambios para racionalizar el proceso de toma de decisiones. El Tratados de Lisboa de 2007 y el Tratado de Funcionamiento de la Unión Europea de 2009 perfeccionaron los anteriores (Matía Sacristan, 2008). El Tratado de Lisboa refuerza la capacidad de actuación de la Unión Europea hacía el exterior y combina las funciones de Alto Representante de la Unión para Asuntos Exteriores y Política de Seguridad con las de Vicepresidente de la Comisión. La base jurídica de la PESC se encuentra en el TUE. Los artículos 21 a 46 del título V del TUE definen las «Disposiciones generales relativas a 
la acción exterior de la Unión y disposiciones específicas relativas a la política exterior y de seguridad común». Los artículos 205 a 222 de la quinta parte del Tratado de Funcionamiento de la Unión Europea (TFUE) tratan de la acción exterior de la Unión También son de aplicación los artículos 346 y 347 de la séptima parte.

Históricamente, Oriente Medio, al Shark al Awsat, Middle East, ha ocupado la atención del mundo más que ninguna otra región. En 1902, Alfred T. Mahan empleó por primera vez el término 'Oriente Medio' para identificar una zona indefinida alrededor del Golfo Pérsico, pero fue a finales de 1930 cuando los británicos reforzaron la aplicabilidad de esta designación al usar el término 'Comando Oriente Medio' para sus fuerzas militares en la zona, que se extendía desde el Mediterráneo central hasta el subcontinente indio. Gradualmente, la aplicabilidad del término fue aceptada después de que la zona quedara bajo la responsabilidad del Comando Oriente Medio (Roucek, 1976, p. 5).

Tras la Primera Guerra Mundial, y la partición del Imperio Otomano, emerge un nuevo mapa, resultante del acuerdo Sykes-Picot y de la colonización europea, que marcó el panorama geopolítico de Oriente Medio. Desde entonces, potencias europeas, Estados Unidos y la URSS han intervenido en la zona por intereses diversos. La compleja descolonización, la difícil formación de los Estados y la perpetuación de sistemas autoritarios, la creación del Estado de Israel, y la interferencia de las grandes potencias en las políticas regionales han marcado decisivamente los acontecimientos regionales del siglo XX. El peso de Oriente Medio en Europa no solo es un producto de siglos pasados, sino también del presente y probablemente del futuro. Oriente Medio es, antes que nada, el vecino de Europa, y éste es un hecho ineludible. Efectivamente, tanto en el pasado como en la actualidad, los acontecimientos ocurridos en Oriente Medio dejaron y dejan su reflejo en Europa, de igual modo se puede afirmar que los acontecimientos ocurridos en Europa dejan sus huellas en Oriente Medio. Los países europeos poseen unas profundas raíces históricas que los han ligado a la zona. Desde sus primeros intereses coloniales de principios del siglo XX, Europa no ha dejado de intervenir, directa e indirectamente, en el proceso de configuración política de Oriente Medio. Las dos guerras mundiales tuvieron una fuerte repercusión en la transformación del mapa político de la zona; así como el desarrollo de la Guerra Fría y el juego estratégico de las potencias occidentales para conseguir una mayor influencia y mantener el control sobre objetivos estratégicos en la región.(Muñoz Fontela, 1992). Para la Unión Europea Oriente Medio es clave en la política mundial y ejerce un impacto directo en ella.

El objetivo de este artículo es proporcionar una visión general de la política exterior de la Unión Europea hacía algunas de las principales cuestiones de Oriente Medio. 


\section{La Unión Europea y el conflicto palestino-israelí}

El conflicto palestino-israelí, también llamado conflicto de Oriente Medio, es, en su plano político, un producto de la Primera Guerra Mundial, es decir, el reparto del último imperio universal oriental, la Turquía Otomana por las potencias occidentales. La Primera Guerra Mundial acabó con el dominio turco en la región. El acuerdo Sykes-Picot, un proyecto colonial pactado entre Francia y Gran Bretaña, establece y determina el reparto de la zona entre ambas potencias. Realizado el reparto, Palestina quedó bajo control británico. Gran Bretaña hizo otra promesa al movimiento sionista para crear un "hogar nacional judío" en Palestina, es la Declaración Balfour. En 1947 las Naciones Unidas abordaron la cuestión de Palestina con el fin de lograr una solución al conflicto. La Asamblea General, el 29 de noviembre de 1947, aprobó la Resolución 181, el plan de partición de Palestina en dos Estados. La Resolución fue rechazada categóricamente por los árabes que impugnaron la competencia legal de la ONU para recomendar la partición de Palestina. La proclamación del Estado de Israel motivó la breve guerra de 1948 que no solucionó ninguno de los problemas básicos del conflicto, pero tuvo como principal consecuencia el establecimiento del estado de Israel sobre el territorio palestino.

Desde su creación, la CEE ha seguido de cerca el desarrollo de los acontecimientos relativos al conflicto palestino-israelí, un conflicto que a su vez marcaba determinadas políticas de la CEE y lo sigue haciendo en la agenda política de Unión Europea. Se trata de uno de los primeros conflictos internacionales en los que la Comunidad se implicó y la necesidad de coordinar las posturas de los distintos Estados miembros que la integran, debido a la importancia de sus intereses desiguales.

La guerra de 1967 había demostrado la falta de cohesión entre las políticas europeas comunitarias. Las distintas posiciones de los Estados miembros reflejan la falta de una política común. Mientras Francia e Italia condenaban a Israel y apoyaban a los árabes, Alemania y Holanda apoyaban a Israel. La guerra de 1973 y el embargo de petróleo contra los países de la CEE, utilizado como medida de presión política, llevó a la CEE a revisar sus políticas respecto al conflicto. La Declaración de Venecia de 1980 reconocía el derecho a la seguridad y a la existencia de todos los Estados de la región, incluido Israel, y la necesidad de cumplir los derechos legítimos del pueblo palestino.

"On the bases thus set out, the time has come to promote the recognition and implementation of the two principles universally accepted by the international community: the right to existence and to security of all the States in the region, including Israel, and justice for all the peoples, which implies the recognition of the legitimate rights of the Palestinian people" "A just solution must finally be found to the Palestinian problem, which is not simply one of refugees. The 
Palestinian people, which is conscious of existing as such, must be placed in a position, by an appropriate process defined within the framework of the comprehensive peace settlement, to exercise fully its right to self-determination" (Council, 1980). La Declaración de Venecia refleja un importante cambio en la postura de la CEE respecto al conflicto palestino-israelí. Desde entonces, la CEE trató de ser un actor más activo en el conflicto de Oriente Medio. "From 1980, the European states decided to take the responsibility to play a direct and active role in seeking a solution. Therefore, the European Union has initiated a set of programs for economic and political cooperation with the states within Middle East, especially with Israel and the Occupied Palestinian Territory but also with Syria, Lebanon, Jordan and Egypt. The EU's consistent support since 1980 for a negotiated two-state solution as the basis for a final and comprehensive settlement of the Israeli-Palestinian conflict has subsequently gained the adherence of the majority of the international community and, above all, since 2002, of the US" (Bolborici, 2020, p. 11).

El impacto de la Intifada Palestina suscitó una emoción en la opinión pública europea, aunque sin llegar a motivar cambios de postura en la política de la CE. La proclamación de un Estado palestino independiente (15/11/1988) por parte de la OLP fue acogido con optimismo por parte del mundo árabe, escepticismo en EE.UU. y prudencia en Europa. La CE expresó su postura en el Consejo de Ministros (Bruselas 21/11/1988) calificando como "pasos positivos" los resultados de la Cumbre de Argel y reafirmando su respaldo a la celebración de una conferencia internacional de paz sobre Oriente Próximo. La postura europea reflejaba la división en este tema entre los países mediterráneos y el resto de los socios comunitarios.

En 1991, el gobierno socialista de Felipe González organizó durante su presidencia europea en España - cuya adhesión a la CEE había tenido lugar en 1986- la Conferencia de Madrid para la Paz. . En su discurso, el presidente del consejo de ministros de la CEE, Hans Van Der Broeck, como jefe de la Delegación de la CEE, señaló que "Mientras que tenemos en mente la proximidad geográfica, una amplia herencia histórica compartida, intensas relaciones a lo largo del espectro político, cultural, económico, y los asuntos humanitarios con la gente de Oriente Medio, la Comunidad y sus Estados miembros no pueden sino tener un gran interés en el futuro de la región, con la que comparte tantos intereses, y que están dispuestos a compartir la construcción de la paz" (Mellado, 1994, p. 323). Sin embargo, la Delegación de la CEE estaba en segundo plano debido al protagonismo de las delegaciones de Estados Unidos y de la URSS, los copatrocinadores de la Conferencia de Madrid. Tampoco la Unión Europea pudo jugar un papel más activo a lo largo de las posteriores negociaciones que desembocaron en los Acuerdos de Oslo. 
La Declaración de Berlín de 1999 incluía un compromiso explícito de los Jefes de Estado y de Gobierno de la Unión Europea con la creación de un Estado palestino "reiteran su apoyo al establecimiento de un acuerdo negociado en Oriente Medio que recoja el principio de "paz por territorios" y garantice la seguridad, tanto colectiva como individual, de los pueblos israelí y palestino. La Unión Europea también hace un llamamiento a las Partes para que reiteren sus compromisos en relación con los principios básicos establecidos en Madrid, Oslo y posteriores acuerdos, de conformidad con las Resoluciones $n^{\circ} 242$ y 338 del Consejo de Seguridad de las Naciones Unidas. La Unión Europea insta a las Partes a que convengan en la ampliación del periodo transitorio fijado en los acuerdos de Oslo. La Unión Europea reitera el derecho constante e incondicional de los palestinos a la autodeterminación, incluida la posibilidad de crear un Estado (Consejo Europeo de Berlín, Conclusiones de la Presidencia, 1999).

La Declaración de Sevilla de junio de 2002, en su anexo VI, señala que "se puede alcanzar un acuerdo a través de la negociación y sólo de la negociación, cuyo objetivo es poner fin a la ocupación y la pronta creación de un Estado de Palestina democrático, viable, pacífico y soberano sobre la base de las fronteras de 1967, si es necesario con algunos ajustes menores acordados por las partes. El resultado final debería ser la existencia de dos Estados que convivan en paz dentro de fronteras seguras y reconocidas y que disfruten de relaciones normales con sus vecinos. En este contexto, debería encontrarse una solución justa a la compleja cuestión de Jerusalén y una solución justa, viable y acordada al problema de los refugiados palestinos" (Consejo de la Unión Europea, Bruselas, 24 de octubre de 2002). La Unión Europea apoyó la "hoja de ruta" como el mejor camino hacia la consecución de la paz, a pesar de que el tiempo transcurrido y la realidad de la situación han llevado a buscar nuevas soluciones.

En 2007, la Unión Europea apoyó activamente el "proceso de Annapolis" que comprometió a los israelíes y a los palestinos a cumplir las obligaciones de la hoja de ruta y a alcanzar un acuerdo de paz a finales de 2008. La Estrategia de Acción de la Unión Europea para la Paz en Oriente Medio de noviembre de 2007 estableció una serie de formas en que la Unión Europea puede apoyar el proceso de paz, incluida la asistencia palestina para la creación de Estado y la resolución global de conflictos sobre la base de la Iniciativa de Paz árabe. "Only an independent, democratic and viable Palestinian state can be a reliable neighbour for Israel. The EU's commitment will therefore focus on assisting Palestinian state building efforts as well as providing support for the transition period. We will strengthen ongoing activities and adjust our priorities in light of developments in the political process, working in partnership with the Palestinian Authority government. The results of the Annapolis meeting and the kind of process it will set in motion will determine the pace and the extent of these adjustments" (Arab League, 2007). 
El 28 de enero de 2020, el presidente Trump presentó su plan llamado "Acuerdo del Siglo" para la reconciliación entre Palestina e Israel Las autoridades palestinas rechazaron la propuesta de Trump descalificándola plenamente. La ONU y otras organizaciones internacionales han cuestionado la forma y el fondo del plan Trump. La Unión Europea, su ministro de Relaciones Exteriores, Josep Borrell, rechazó la iniciativa y reiteró la posición de la Unión Europea, según la cual la organización se opone a la existencia de asentamientos en Judea y Samaria, en Jerusalén Oriental y en los Altos del Golán. "La Unión Europea no reconoce la soberanía israelí sobre los territorios ocupados en 1967. No nos quedaremos de brazos cruzados si Israel lleva a cabo medidas para anexarlos." Borrell llamó a las partes del conflicto a resolver los problemas relacionados con el estatus final mediante "negociaciones directas" para "construir una paz justa y duradera". Borrell, de este modo, se ha referido a la necesidad de una solución de dos Estados para el conflicto palestino-israelí, con base en las fronteras de 1967 y que tenga a Al-Quds (Jerusalén) como su capital. El responsable de la Política Exterior de la Unión Europea reiteró que la Unión Europea respalda la solución de dos Estados. La posición de Unión Europea mantiene su compromiso con la solución de los dos Estados.

\section{La Unión Europea y las relaciones euromediterráneas}

Desde el comienzo del diálogo euro-árabe, los países de la CEE han sido conscientes de que la preocupación esencial de los países árabes era de tipo político, mientras que ellos han dado una mayor importancia a los problemas económicos. Sin embargo, desde el fin de la guerra fría, la CEE ha adoptado una serie de medidas y declaraciones que favorecen la efectividad de la llamada "alternativa europea", frente a la política estadounidense en la región. El éxito de esta alternativa depende en gran medida de la implicación de Europa en las cuestiones político-culturales del Mundo Árabe con nuevos enfoques y verdaderas perspectivas de futuro (Abu-warda, 2000).

Todo empezó cuando en noviembre de 1973, en la Conferencia Cumbre de la CEE de Bruselas, una declaración conjunta en la que los miembros de la organización expresaron su postura sobre la crisis de Oriente Medio, postura que se resumía en los siguientes puntos: condenar la adquisición israelí de los territorios ocupados en 1967, respetar los legítimos derechos del pueblo palestino, y respetar la independencia, la soberanía y la integridad territorial de todos los países de la región. La respuesta árabe surgió en la $6^{\mathrm{a}}$ Cumbre Árabe de Argel en noviembre de 1973, proponiendo la idea de un diálogo mutuo euroárabe 
En marzo de 1974, se celebró en París una conferencia de parlamentarios europeos que dio lugar al nacimiento de la Asociación Parlamentaria para la Cooperación Euro-Árabe (A.P.E.C.A.). A nivel árabe, la Resolución 3123 del Consejo de la Liga Árabe anunció la creación de un comité árabe para el diálogo con la CEE y en 1974 se creó la Unión Interparlamentaria Árabe (U.I.P.A.). La Cumbre Árabe de Rabat de 1974 aceptó el principio del diálogo euro-árabe.

La primera sesión de la Comisión General tuvo lugar en Luxemburgo en mayo de 1976. En el comunicado final ambas partes renuevan su punto de vista sobre el conflicto árabe-israelí y reconocen los legítimos derechos del pueblo palestino. La segunda reunión (Túnez 10-11 de febrero 1977) destaca por sus grandes avances en el campo político y en el económico. Políticamente, su comunicado final recoge de nuevo los legítimos derechos del pueblo palestino, y por primera vez, afirma su oposición a la política israelí en los territorios ocupados.

En la Declaración de París (26-3-79) la postura de la CE oscila entre dos sensibilidades: por una parte, exigen en el tratado de Camp David una correcta aplicación de la resolución 242, y, por otra, expresan su deseo de que el Tratado lleve a una solución global en la que sean contemplados los derechos del pueblo palestino. Sin embargo, el acontecimiento más importante en la postura comunitaria, con relación al conflicto árabe-israelí, vino de Venecia (junio 1980).

La década de los ochenta se caracteriza por una serie de acontecimientos regionales e internacionales de especial importancia y que incidieron negativamente en el diálogo interregional, dificultando su avance y limitando sus resultados.

En la Cumbre Europea de Rodas, 2-3 de diciembre de 1988, los doce acordaron promover una conferencia internacional de paz en la región árabe. Durante la presidencia española de la CE, la política de la troika comunitaria en relación con Oriente Medio destaca por sus intentos de impulsar al máximo iniciativas de paz. Con el fin de reactivar el diálogo euro-árabe, y a propuesta del presidente francés François Mitterrand, el Parlamento europeo organizó una Conferencia ministerial euro-árabe en París, en diciembre de 1989, encargada de impulsar el diálogo entre las dos regiones y activar la cooperación. De la Conferencia de París surgen dos grupos de trabajo, uno de carácter económico y social y el otro encargado de la reestructuración del diálogo euro-árabe. La Conferencia de París tenía la posibilidad de revitalizar el diálogo euro- árabe. Sin embargo, los acontecimientos regionales e internacionales que siguieron a la Conferencia eclipsaron las relaciones y el diálogo.

Con el fin de corregir las deficiencias del diálogo y de la "política global mediterránea", la CE aprobó una nueva política a seguir en sus relaciones con los países mediterráneos no comunitarios: la "Política Mediterránea Renovada". La 
Política Mediterránea Renovada, consiste en "renovar" y reforzar los vínculos y relaciones de cooperación con los países mediterráneos con el fin de evitar el deterioro de las balanzas comerciales de dichos países. Dentro del marco general de la PMR, el Consejo Europeo de Lisboa (junio 1992) aprobó un proyecto de Asociación Euro-Magrebí que comprende una cooperación descentralizada, programas mediterráneos (MED-CAMPUS, MED-URBS, MED-INVEST, MED-MEDIA), así como el compromiso de la Unión Europea de ayudar a la reconstrucción de la economía palestina y promover la integración regional de Oriente Próximo. Este proceso de aproximación Euro-Mediterránea se ha visto reforzado por otros proyectos o fórmulas de cooperación como el Grupo 5+5, la CSCM, la Asociación Euro-Mediterránea y la Conferencia Euro- Mediterránea de Barcelona.

Una serie de acontecimientos marcaron la geopolítica del Mediterráneo a lo largo de 1991: la Guerra del Golfo, la Conferencia de Paz árabe- israelí, Argelia, el Proyecto STS, el diálogo CE-UMA (iniciado en 1990), etc. Con el fin de reactivar los contactos, la iniciativa hispano-italiana, presentada en la Conferencia de Seguridad y Cooperación en Europa (CSCE) (septiembre 1990), de convocar otra similar en el espacio mediterráneo, fue una de las propuestas de mayor éxito en aquellos años para la cooperación euro-árabe.

La Conferencia se plantea como un medio de sustituir la confrontación por la cooperación en las relaciones entre las dos orillas del Mediterráneo. Para conseguir dicho objetivo, fue convocada en Málaga la CSCM, a nivel parlamentario, del 15 al 20 de junio de 1992. Entre las principales cuestiones planteadas como objetivos a realizar destacan: la seguridad y estabilidad en la región, un desarrollo económico y social equilibrado, la creación de un marco de convivencia pacífica en la región, el intercambio cultural, y la construcción de un espacio de relaciones de buena vecindad. Otra iniciativa similar ha sido el "Foro Mediterráneo" nacido de un proyecto franco-egipcio y que tuvo su reunión fundacional en Alejandría en julio de 1994. Son algunas iniciativas de diálogo informal entre los países ribereños sobre cuestiones de interés común.

La Conferencia Euro-mediterránea de Barcelona, los días 27 y 28 de noviembre de 1995, vuelve a plantear las grandes cuestiones en las relaciones entre los Estados de la cuenca mediterránea y, en cierto modo, culmina viejos proyectos de cooperación entre ambas orillas, proyectos como el diálogo euroárabe iniciado en la década de los 70, el Grupo 5+5, o la CSCM, etc. La iniciativa contempla la puesta en marcha de una política de desarrollo compartido. Hasta llegar a Barcelona fueron necesarias una serie de decisiones iniciadas en junio de 1992 en el Consejo Europeo de Lisboa con la aprobación de la propuesta de crear una Asociación Euro-Magrebí, más tarde el Consejo Europeo de Corfú (junio 1994) encargó al Consejo y a la Comisión la evaluación de la política global de la Unión Europea en la región del Mediterráneo, así como la realización 
de eventuales iniciativas destinadas a reforzar dicha política a corto o medio plazo. También el Consejo Europeo de Essen (diciembre 1944) se congratula de la intención de la presidencia española de convocar una conferencia euromediterránea con la participación de todos los países mediterráneos interesados². La conferencia deberá permitir que se celebren debates en profundidad sobre las futuras relaciones entre la Unión Europea y los países mediterráneos, en los que se aborden todas las cuestiones políticas, económicas, sociales y culturales. Convocada la Conferencia de Barcelona, los temas debatidos se dividen en tres "cestos" temáticos: política y seguridad, cooperación económica y temas sociales y humanos. El interés de cada uno de los cestos varía de una a otra orilla, mientras los países de la Unión Europea muestran mayor preocupación por los temas políticos y de seguridad, los otros países mediterráneos destacan la importancia del cesto económico. La Conferencia Euro-Mediterránea de Barcelona puede calificarse de un éxito relativo y, a pesar de la no inclusión de Libia en la reunión y los problemas surgidos para la firma de la Declaración Final, lo cierto es que se ha llegado a un consenso.

Tras otras conferencias y consejos, los días 27 y 28 de noviembre de 2005 tuvo lugar la Conferencia Euromediterránea II. los líderes Euromediterráneos se reunieron en Barcelona para celebrar diez años de la adopción de la Declaración de Barcelona. Lo más destacable de la Conferencia es la llamada Política Europea de Vecindad (PEV) que serviría como complemento para el Proceso de Barcelona, así como el reforzamiento de la PESD. La PEV había sido lanzada en 2004 como mecanismo de cooperación inicialmente pensada para los países del Este y sus futuras ampliaciones en el marco de la Unión Europea, con el objetivo de consolidar la estabilidad, la seguridad y el bienestar. La Conferencia Euromediterránea II no tuvo grandes éxitos.

Tras la Cumbre de Barcelona, con el fin de reactivar el proceso, el Gobierno francés declaraba la necesidad de "relanzar y revitalizar" el Partenariado. "En 2007, Nicolás Sarkosy, en campaña presidencial francesa, propuso como objetivo de campaña relanzar la cooperación euromediterránea, estancada en esos momentos, a través de un proyecto titulado: Unión por el Mediterráneo (UpM). Su llegada al poder supuso un cambio radical en las relaciones euromediterráneas, posicionándolas en un lugar primordial en la agenda del nuevo mandatario. Sarkozy afirmaba que el fracaso del Proceso de Barcelona se debía, entre otras cosas, a que era un proceso controlado desde las instituciones europeas, lo que restringía una completa participación de las partes, e instaba a las partes a dar un nuevo impulso de cooperación, un "Mediterráneo para los mediterráneos" (Calderón Hernández, 2016, p.18).

${ }^{2}$ En el foro participaron Egipto, Francia, Argelia, Grecia, Marruecos, Portugal, Túnez, Turquía, Italia, España y Malta. Libia intentó participar en el Foro pero sin conseguirlo, debido al bloqueo impuesto sobre el país.

Araucaria. Revista Iberoamericana de Filosofia, Política, Humanidades y Relaciones Internacionales, año $22, \mathrm{n}^{\circ} 45$. Tercer cuatrimestre de 2020. Pp. 499-526. ISSN 1575-6823 e-ISSN 2340-2199 https://dx.doi.org/10.12795/araucaria.2020.i45.21 
El 13 de julio de 2008 nació la Unión para el Mediterráneo (UpM). Una nueva iniciativa que tiene como finalidad la creación de un área de paz, estabilidad y seguridad basada en una cooperación multilateral con vistas a una integración regional y cohesión. La UpM establece estructuras permanentes, a fin de lograr una mayor institucionalización de sus actividades. La iniciativa Unión por el Mediterráneo es una etapa más en el desarrollo del Proceso de Barcelona. Se compone de 43 países de Europa y de la cuenca mediterránea. Sus principales objetivos consisten en promover la estabilidad y la integración en toda la región mediterránea, mejorar la integración norte-sur y sur-sur en la región mediterránea para así potenciar el desarrollo socioeconómico de los diferentes países que la componen y asegurar su estabilidad. "Aunque fue lanzada con grandes expectativas, la UpM no ha despegado debido a varios condicionamientos. Desde el punto de vista político, pronto se ha transformado en un rehén del conflicto entre Israel y Palestina, con los gobiernos árabes que, tras la operación israelí en Gaza bloquearon los trabajos de la nueva estructura, mientras que la Libia de Gadafi rechazó entrar y la Unión encontró dificultades inmensas a la hora de individualizar a una personalidad que pudiera ser el Secretario General, como lo demuestran los nombramientos sucesivos entre 2010 y 2011" (Guasconi, 2013, p. 146).

Tras la revisión de la Política europea de Vecindad y las reuniones de ministros de Asuntos Exteriores de la UpM de noviembre de 2015 y enero de 2017 en Barcelona, la UpM entra en una fase en la que, una vez consolidada, se presenta como el mejor espacio para el desarrollo de las relaciones euromediterráneas y el marco multilateral de la política europea de vecindad para el Sur, complementario de las relaciones bilaterales de la Unión Europea con estos países así como para la coordinación de iniciativas en el ámbito mediterráneo ${ }^{3}$.

Desde su creación, la Unión Europea, ha tenido siempre en cuenta a sus vecinos del sur del Mediterráneo. El objetivo de estas políticas siempre ha sido el mismo: la estabilidad de la zona, un objetivo sin alcanzar a pesar de todas las iniciativas y procesos mencionados. El objetivo es suficientemente claro, sin embargo, los procesos para alcanzarlo son menos claros. El poco éxito de tantas iniciativas llama a la reflexión sobre las políticas utilizadas en ambas orillas del Mediterráneo.

\footnotetext{
${ }^{3}$ http://www.exteriores.gob.es/Portal/es/PoliticaExteriorCooperacion/Mediterraneo/Paginas/ PartenariadoEuroMediterraneo.aspx.
}

Araucaria. Revista Iberoamericana de Filosofí, Política, Humanidades y Relaciones Internacionales, año $22, \mathrm{n}^{\circ} 45$. Tercer cuatrimestre de 2020. Pp. 499-526. ISSN 1575-6823 e-ISSN 2340-2199 https://dx.doi.org/10.12795/araucaria.2020.i45.21 


\section{La Unión Europea y el Consejo de Cooperación del Golfo}

La subregión del Golfo, como zona de Oriente Medio, es una región de primer interés geopolítico y económico para la Unión Europea. El Consejo de Cooperación del Golfo, (CCG), creado en mayo de 1981, está compuesto por Baréin, Kuwait, Omán, Qatar, Arabia Saudí y los Emiratos Árabes Unidos. Sus objetivos consisten en la creación de una Unión Aduanera y una cooperación más estrecha en el sector monetario, así como en la energía, las inversiones y el desarrollo económico.

La Unión Europea y el CCG han adoptado posiciones comunes sobre cuestiones relacionadas con Oriente Próximo. La importancia de las relaciones UE-CCG reside en su alto grado de interdependencia energética y económica. La Unión necesita la energía suministrada por los países del CCG, que encuentran en los Estados miembros un gran mercado que absorbe una parte importante de sus exportaciones de productos petrolíferos refinados, productos petroquímicos y aluminio. La interdependencia económica entre la Unión Europea y el CCG es muy estrecha. Por orden de importancia, el CCG es el quinto mercado para las exportaciones de la Unión y el único de los cinco en el que la Unión viene registrando desde siempre un excedente comercial. Por otro lado, los países del CCG han seguido desempeñando un papel activo en el contexto de la diplomacia de Oriente Próximo.

La CEE y el CCG firmaron un primer Acuerdo de cooperación en 1988. $\mathrm{Su}$ objetivo es reforzar la estabilidad en una región de importancia estratégica, facilitar las relaciones políticas y económicas y reforzar la cooperación económica y técnica, así como estrechar la colaboración en materia de energía, industria, comercio y servicios, agricultura, pesca, inversiones, ciencia, tecnología y medio ambiente. El Acuerdo contempla la celebración de consejos conjuntos y reuniones a nivel ministerial, así como la constitución de comités mixtos de cooperación compuestos por altos cargos. El Acuerdo no prevé ningún órgano de carácter parlamentario. La prioridad de los europeos en aquella época era asegurarse sobre todo sus importantes necesidades de suministro energético y, como consecuencia de ello también un precio estable para dicho sector. Mientras, las aspiraciones de la otra parte, los países del Golfo, consistían en obtener unas regulaciones más favorables para sus exportaciones de petróleo y otras materias (Echagüe, 2007).

En 1995, la Comisión sugirió tomar medidas que fortalecieran las relaciones entre la Unión Europea y el CCG, entre las cuales se encontraban el establecimiento de un diálogo político. En diciembre de 2003, la Comisión y el Alto Representante para la Política Exterior y de Seguridad Común (PESC) presentaron un documento de políticas que hacía hincapié en la necesidad de extender y profundizar el diálogo entre la Unión Europea y el CCG, y relacionar los marcos de la UE-CCG y la Alianza Euromediterránea. 
La Comisión Europea, en un comunicado de prensa, 22 de noviembre de 1995, sobre las relaciones entre la Unión Europea y los países del Consejo de Cooperación del Golfo, informó sobre las conclusiones de una reunión ministerial en forma de troika con ministros del CCG, señalando que: Se refuerce el diálogo político entre la Unión Europea y el CCG; que se intensifique la cooperación económica y se propongan soluciones al actual bloqueo de las negociaciones para la celebración de un acuerdo de libre cambio; que se elaboren instrumentos de cooperación, en particular en los ámbitos cultural y científico, que permitan a las dos partes conocerse mejor y comprenderse más. El comunicado hace referencia al diálogo político entre ambas organizaciones, ya que los países de la Unión Europea han desempeñado un importante papel en la seguridad del Golfo y además el refuerzo del diálogo político permitirá también a la Unión Europea y al CCG medir y coordinar de manera más acertada su apoyo al proceso de paz en Oriente Medio, y discutir los problemas internacionales de interés común. Para reforzar el diálogo político, el proyecto de comunicación propone la organización de reuniones semestrales de altos funcionarios.

Tras el 11 de septiembre de 2001 aparecieron nuevas propuestas en el terreno de la seguridad como la Estrategia Europea de Seguridad, la Iniciativa de Cooperación de Estambul (ICE) y otras iniciativas. Se acordó definir un rol de seguridad en la región del Golfo y se organizó un taller UE-CCG sobre terrorismo. En el ámbito de la seguridad, los Estados del CCG no han logrado, o no han querido forjar, una política de defensa y seguridad común. Los Estados del Golfo dan prioridad a las amenazas a la seguridad del régimen. El objetivo principal de su política exterior es la seguridad del régimen, la cual es el paradigma que define la política. La política exterior está impulsada por las preocupaciones en materia de seguridad interna. Los logros más significativos han sido hasta el momento la creación del Península Shield Force (fuerza militar conjunta de alrededor de 15.000 efectivos) en 1982; el compromiso en 1997 de unir los Estados con una red de comunicación militar de alerta rápida; y la formación de un Consejo de Defensa Conjunto refrendado en 2001. La razón fundamental es la creciente fisura entre Qatar y Arabia Saudí, así como la existencia de acuerdos de defensa con EE. UU., el Reino Unido y Francia y la mayor prioridad otorgada a la seguridad interna. Estados Unidos ha oficiado de "garante de la seguridad" en el Golfo desde la década de 1970. (Echagüe, 2007, p. 20). Sin embargo, los regímenes del Golfo no tienen certeza ni la confianza en los EE.UU. Además, su opinión pública cuestiona la política de los EE.UU. en el Golfo. En materia de seguridad, la Unión Europea no puede o no quiere competir con los EE.UU. Bilateralmente, Francia y el Reino Unido mantienen acuerdos de defensa. Desde la década de 1990, la venta de armas a la región proveniente de países de la Unión Europea, Reino Unido, Francia, España e Italia se ha incrementado significativamente ${ }^{4}$.

${ }^{4}$ En diciembre del 2005, el Reino Unido firmó un acuerdo con Arabia Saudí para suministrar 72 
La Unión Europea y el CCG acordaron un programa de acción conjunta para el periodo 2010-2013 en el que establecieron una hoja de ruta para reforzar la cooperación en, entre otros ámbitos, las tecnologías de la información y la comunicación, la seguridad nuclear, las energías limpias, la investigación y el diálogo económico. El 24 de marzo de 2011 el Parlamento aprobó una Resolución sobre las relaciones de la Unión Europea con el CCG en la que se pronunciaba a favor del establecimiento de una asociación estratégica con el CCG. Esta posición se reiteró en la Resolución del Parlamento Europeo, de 9 de julio de 2015.

Desde 2015, la Unión Europea y el CCG han participado en conversaciones sobre los desafíos que enfrenta la diversificación económica en la región del Golfo. En abril de 2016, el Comité Mixto de Cooperación UE-CCG acordó establecer un diálogo informal más estructurado en materia de comercio e inversión. En julio de 2016, se celebraron un consejo conjunto y una reunión ministerial entre la Unión Europea y el CCG. Como consecuencia del conflicto entre Qatar y Arabia Saudita, que estalló en 2017, no se han convocado nuevas reuniones.

En los últimos años, el Parlamento ha aprobado distintas resoluciones en las que se muestra preocupado por la situación de los derechos humanos en los países del Golfo. Especialmente, con la guerra de Yemen, el Parlamento ha pedido en varias ocasiones que se imponga un embargo de armas de la Unión Europea a Arabia Saudí, por las graves acusaciones de violaciones del Derecho internacional humanitario en Yemen. Sin embargo, los Estados del CCG han bloqueado la creación de un diálogo formal con la Unión Europea; las iniciativas de la Unión Europea hacia la sociedad civil son rechazadas; los proyectos de gobernanza tampoco son aceptados. Los Estados del Golfo se han resistido a abrirse a la Unión Europea para cooperar con la reforma política y promoverla; su interés en tener una asociación estratégica más amplia excluye cualquier escrutinio de la situación política interna. La misma Unión Europea reconoce tener dificultades a la hora de encontrar puntos de acceso que le permitan apoyar proyectos de la sociedad civil como lo hace en otros lugares (Echagüe, 2007, p. 23).

El refuerzo del diálogo político entre la Unión Europea y el CCG encuentra su razón de ser en los intereses políticos y de seguridad que comparten. Los países de la Unión Europea han desempeñado un importante papel en la seguridad del Golfo durante las guerras del Golfo. La Unión Europea debería confirmar el interés que en ella sigue despertando la seguridad del CCG en el marco de su política exterior y de seguridad común. El refuerzo del diálogo

aviones de combate Eurofighter Typhoon con los que reemplazar los Tornados y otros en servicio al momento en la Fuerza Aérea saudí. Se dice que el negocio costó alrededor de 60.000 millones de euros, e incluye en el contrato el entrenamiento, repuestos y reparaciones. 
político permitirá también a la Unión Europea y al CCG coordinar de manera más acertada su apoyo al proceso de paz en Oriente Medio y discutir los problemas internacionales de interés común.

\section{La Unión Europea ante la "Primavera Árabe"}

Desde 2011, las movilizaciones políticas, impulsadas por la falta de libertad y democracia en muchas de las naciones árabes, anunciaban el fin de una era y el comienzo de nuevos tiempos. La Primavera Árabe, si bien partió como protestas populares, en varios países, el alzamiento civil tomó un protagonismo global. En Túnez y en Egipto las movilizaciones derrocaron a los respectivos gobiernos en pocas semanas, pero en países como Libia o Siria, el gobierno respondió violentamente y se negó a abandonar el poder, lo que llevó a la población a armarse y a comenzar guerras civiles en ambos países. En Libia, los rebeldes insurgentes derrocaron a las fuerzas gubernamentales seis meses después del inicio de la revolución. Sin embargo, en Siria, la guerra se ha prolongado y el impacto bélico intensificado, sin visualizarse un fin cercano y con registros que estiman en cientos de miles la cifra de muertos.

El Tratado de Lisboa (2007) permite a la Unión Europea tener una Política Común de Seguridad y Defensa que, entre otras cosas, pretendía reforzar su papel de actor en la esfera internacional. Como otros actores internacionales, la Unión Europea tiene una postura y una política especifica hacía los acontecimientos en cada uno de los escenarios y países árabes ${ }^{5}$.

Al comienzo, el apoyo a la revolución árabe llegaba de diferentes partes del mundo. Los informes de la ONU señalaban que la mayoría de las protestas era de naturaleza pacífica, y que las fuerzas de seguridad habían reaccionado con una fuerza excesiva que no cumplía con los estándares internacionales. La Unión Europea, su jefa de Política Exterior, Catherine Ashton expresó su apoyo y reconocimiento al pueblo tunecino y sus aspiraciones democráticas, que deben ser alcanzadas de manera pacífica. La Unión Europea también expresó su disposición a ayudar a encontrar soluciones democráticas a la crisis en curso. Sin embargo, la intervención directa de las grandes potencias internacionales (la OTAN, EEUU, Rusia, Reino Unido, Francia, Unión Europea, etc.), motivadas por intereses geopolíticos, estratégicos o económicos, ha permitido ciertos cambios formales en algunos países, pero sin cambios reales importantes. En otros casos, Libia y Siria, los objetivos de las intervenciones extranjeras son estructurales y más profundos, como neutralizar los últimos focos de

${ }^{5}$ El Tratado de Maastricht establece transforma la CCE en la Unión Europea y crea el pilar de la Política Exterior y de Seguridad Común (PESC), con el objetivo del "mantenimiento de la paz y el fortalecimiento de la seguridad internacional". 
resistencia a la existencia del Estado de Israel, al panarabismo y al sueño de una "nación árabe unida". Un proyecto viejo, que no está concluido, comenzó en Egipto, con los Acuerdos de Camp David, normalizando las relaciones entre Egipto e Israel, neutralizar Egipto en la guerra árabe-israelí y acabar con el panarabismo de Nasser. La invasión de Irak en 2003 tenía como principal objetivo desmantelar al panarabismo bathista. La guerra en Libia y en Siria es otra etapa en el viejo-nuevo proyecto que pretende dividir aún más un mundo árabe fragmentado y debilitado por las potencias aliadas de la primera Guerra Mundial.

Las primeras reacciones europeas frente a las protestas árabes se han caracterizado por la sorpresa y la cautela con las que los gobiernos y las instituciones de Bruselas han manifestado su apoyo a estos movimientos. Mientras en el mundo árabe los jóvenes luchaban por su libertad y la sociedad pedía democracia y respeto a los derechos, Europa parecía incapaz de tomar la iniciativa y asumir el papel que la historia y su posición le solicitarían; mejor dicho, su respuesta ha sido «lenta, débil, dividida e incoherente», como lo ha demostrado el hecho de que la primera reacción concreta de la Unión se dio el 21 de marzo de 2011, cuando el Consejo congeló los bienes del dictador egipcio Hosni Mubarak (Guasconi, 2013, p.135).

En todas las manifestaciones, el conflicto libio y la guerra de Siria representan los modelos más dramáticos de la Primavera Árabe. En ambos Estados las movilizaciones populares fueron violentamente reprimidas y pese a que crímenes de guerra, crímenes contra la humanidad y grandes flujos de refugiados son elementos comunes a ambas, la respuesta de la sociedad internacional ha sido muy diferente (Cervell Hortal, 2014, p. 2).

En Libia la población civil comenzó a exigir cambios políticos. Las primeras manifestaciones fueron entre enero y febrero de 2011, provocando la inmediata reacción del gobierno: represión y violencia. Las fuerzas leales del gobierno comenzaron a luchar contra los opositores, quienes habían creado el Frente de Liberación de Libia. La violencia se propagó por todo el país, y en la mayoría de las ciudades se desarrollaban combates entre los rebeldes y el ejército libio de Gadafi. Tras fuertes combates, lo que había iniciado como una manifestación terminó en una guerra civil.

El 19 de marzo, políticos de la Unión Europea y la Liga Árabe, entre otros, deliberaron en París sobre el procedimiento a seguir en Libia. La resolución 1973 del Consejo de Seguridad (2011) abrió la puerta al uso de la fuerza con el único fin, según se recogía, de proteger a los civiles, pero excluyendo de manera expresa la presencia de "una fuerza de ocupación extranjera de cualquier tipo en cualquier parte del territorio libio". Una coalición de aliados de la OTAN y otros países árabes comenzaron una operación cuya finalidad se decía que era la ejecución de un embargo de armas, mantener una zona de exclusión 
aérea y proteger a los civiles. Estados Unidos, Francia y Reino Unido iniciaron los primeros ataques militares a Libia. Tras ataques aéreos masivos contra instalaciones militares libias, los aliados imponían la exclusión aérea. Los bombardeos en contra del gobierno y de sus instalaciones colapsaron al Estado e hicieron que Gadafi perdiera el control del país y se vio en la necesidad de huir. Los rebeldes, en tanto, habían avanzado por todo el país. El 20 de octubre de 2011, Gadafi fue detenido y ejecutado (Abu-warda, Najib and Rubio Garcia, D. 2018, p. 301).

Tras una declaración de la alta representante de la Unión Europea para Asuntos Exteriores y Política de Seguridad, Catherine Ashton, en la que se expresaba la preocupación por la situación en Libia y se pedía restricción del uso de la fuerza, la Comisión aprobó el establecimiento de un mecanismo de protección civil y asistencia humanitaria, mientras el Consejo acordó imponer al régimen libio nuevas sanciones y restricciones. En la sesión plenaria de marzo, el PE tomó la iniciativa de recibir en Estrasburgo a una delegación del Consejo Nacional de Transición (CNT) antes de que éste fuera reconocido por ningún Estado Miembro como único representante legítimo de Libia. Numerosos europarlamentarios reclamaron entonces el reconocimiento de la organización rebelde por parte de la Unión Europea, la salida del poder de Gadafi y la imposición de una zona de exclusión aérea (Stavridis, 2013, p. 16).

La operación EUNAVFOR MED SOPHIA se puso en marcha el 22 de junio de 2015 y forma parte del enfoque global de la Unión Europea en materia de migración. La misión es parte de un plan más amplio de la Unión Europea para gestionar las oleadas migratorias. La operación tiene también una serie de tareas de apoyo: proporciona formación a la guardia costera y la Armada libias, supervisa la eficiencia a largo plazo de la formación y contribuye a la aplicación del embargo de armas de las Naciones Unidas en alta mar frente a las costas de Libia. Además, la operación también realiza actividades de vigilancia y recoge información sobre la exportación ilícita de petróleo crudo de Libia, de conformidad con las resoluciones del Consejo de Seguridad de las Naciones Unidas.La operación contribuye a los esfuerzos de la Unión Europea por devolver la estabilidad y la seguridad a Libia, así como a la seguridad marítima a la región del Mediterráneo central (Consejo Europeo 2016). La Unión Europea intensificando sus esfuerzos para aplicar el embargo de armas de las Naciones Unidas a Libia, contribuyendo así al proceso de paz en este país, mediante la puesta en marcha de una nueva operación militar de la PCSD (política común de seguridad y defensa) en el Mediterráneo, el 31 de marzo de 2020, el Consejo puso en marcha la operación IRINI con el objetivo principal de hacer cumplir el embargo de armas de las Naciones Unidas en Libia (Release, 2020) ${ }^{6}$.

${ }^{6}$ Decisión (PESC) 2020/472 del Consejo de 31 de marzo de 2020 relativa a una operación militar de la Unión Europea en el Mediterráneo (EUNAVFOR MED IRINI).

Araucaria. Revista Iberoamericana de Filosofia, Política, Humanidades y Relaciones Internacionales, año $22, \mathrm{n}^{\circ} 45$. Tercer cuatrimestre de 2020. Pp. 499-526. ISSN 1575-6823 e-ISSN 2340-2199 https://dx.doi.org/10.12795/araucaria.2020.i45.21 
El 19 de enero de 2020 tuvo lugar la Conferencia de Berlín sobre Libia. Se trataba de apoyar los esfuerzos de la ONU para lograr una tregua y una vía para una solución política dentro de Libia. A la Unión Europea le interesa la paz en Libia por su potencial energético, pero también porque es una de las rutas migratorias principales de África a Europa. Las conclusiones de la conferencia otorgan un amplio mandato para complacer a todos los participantes, pero no proporcionan soluciones para romper el estancamiento de la guerra libia. En su Declaración, el alto representante de la Unión Europea señala que la Unión Europea pide a todas las partes que actúen con responsabilidad y cesen de inmediato los combates en todo el territorio libio y recuerda a las partes que tienen la obligación de respetar el Derecho internacional, incluido el Derecho internacional humanitario. La Unión Europea hace un llamamiento a todas las partes en el conflicto de Libia para que cooperen sin más demora con objeto de hacer efectivo un alto el fuego duradero, confirmando lo antes posible su acuerdo sobre el proyecto debatido en el Comité Militar Conjunto celebrado en Ginebra el 23 de febrero de 2020. La tregua debe ir a la par con la inmediata reanudación de las conversaciones entre las partes, auspiciadas por las Naciones Unidas, y respetando plenamente el Acuerdo Político Libio. No hay alternativa a una solución política integradora que refleje las conclusiones de la Conferencia de Berlín, confirmadas en la Resolución n 2510 del CSNU. La Unión Europea reitera su compromiso con la soberanía, la unidad y la estabilidad de Libia y con el Derecho internacional (Release, 2020). Con la operación IRINI, la Unión Europea logra mantener a Europa presente y activa en Libia, como la potencia que está tratando públicamente de hacer cumplir las normas diplomáticas, y responsabilizando a los beligerantes de todo tipo, es la única forma de reinsertarse en la ecuación libia. Sin embargo, diez años después de Gadafi, Libia sigue siendo un Estado fallido, abandonado a su suerte en una guerra civil sin sentido.

El conflicto sirio, que enfrenta al gobierno junto a las Fuerzas Armadas de Siria en contra de diversos grupos de la oposición que buscan derrocar al gobierno y, en particular, sacar del poder al presidente Bashar Al-Asad, es un conflicto asimétrico y constante sin perspectivas de solución a corto plazo. La crisis comenzó a inicios de 2011 cuando parte de los sirios comenzó a alzarse en una serie de protestas en contra del gobierno. Los manifestantes reclamaban más libertades y plena democracia, así como también un mayor respeto de los derechos humanos. La respuesta a las manifestaciones provocó que una parte de la sociedad civil y sectores del Ejército se alzaran en armas formando el llamado «Ejército Libre de Siria», a lo cual se sumaron otros grupos opositores al gobierno. Rápidamente, estos grupos insurgentes fueron tomando ciudades importantes del país. La revolución había degenerado en una guerra civil. Dentro de la Primavera Árabe, la guerra civil siria es la más compleja (Abu- 
warda, Najib and Rubio Garcia, D. 2018, p. 306). La Unión Europea ha adoptado sanciones contra Siria como consecuencia de la violencia contra la población civil y para compensar los fracasos de los proyectos de resolución presentados al Consejo de Seguridad de la ONU. De forma un tanto desafinada y poco planificada, a partir del 9 de mayo de 2011, el Consejo fue aprobando decisiones y reglamentos con muy diversas medidas restrictivas contra Siria y contra las personas responsables de la represión violenta ejercida contra la población civil de Siria.

En 2017, el Consejo adopta la Estrategia de la Unión Europea para Siria. Es parte de la Estrategia regional de la Unión Europea para Siria e Irak, así como en relación con la amenaza que representa el Daesh. Los objetivos estratégicos de la Unión Europea en Siria se centran en seis ámbitos principales: poner fin a la guerra mediante una auténtica transición política; promover una transición constructiva e integradora en Siria; salvar vidas atendiendo a las necesidades humanitarias de los sirios más vulnerables; promover la democracia, los derechos humanos y la libertad de expresión; promover la rendición de cuentas por crímenes de guerra; reforzar la capacidad de recuperación de la población y la sociedad sirias (Council of the EU, 2017) .

En la cuarta Conferencia de Bruselas «Apoyo al futuro de Siria y su región», la comunidad internacional comprometió un total de 6900 millones de euros para Siria y los principales países que acogen a refugiados sirios para 2020 y años posteriores. De este importe, la Unión Europea ha comprometido el 71 \%. La Unión Europea y sus Estados miembros son quienes más aportan para superar las consecuencias de la crisis siria, con más de 20000 millones de euros en concepto de ayuda humanitaria, ayuda a la estabilización, ayuda al desarrollo y ayuda económica, movilizados desde 2011 (UE et al., 2020).

En los dos conflictos expuestos, Siria y Libia, la Unión Europea ha demostrado su limitada capacidad para intervenir con eficacia en la gestión de conflictos internacionales. Su Política Común de Seguridad y Defensa queda cuestionada debido al poco consenso que tienen los miembros de la Unión Europea a la hora de hacer frente a determinadas crisis internacionales. El grueso de las intervenciones en ambos conflictos fue protagonizado por Rusia, Estados Unidos, Turquía e Irán, quedando la Unión Europea en un segundo plano, asegurado su presencia en las conversaciones de paz. Tanto en Siria como en Libia, la Unión Europea ha demostrado que aún es un actor débil, lo que confirma que la PCSD tiene mayor capacidad para gestionar situaciones de post conflicto más que la intervención directa en los conflictos. La limitada capacidad de la Unión Europea para intervenir de modo más eficaz y competitivo con otras potencias internacionales, como los EEUU y Rusia, en los acontecimientos de Oriente Medio, hace necesaria una nueva estrategia y una revisión de la PCSD y de la PESC. 


\section{La postura de la Unión Europea hacía la cuestión nuclear iraní}

El programa nuclear iraní se inició en la década de los años 50 del pasado siglo. En 1957, el Sha firmó un Acuerdo con Estados Unidos relativo a la cooperación en la investigación del uso pacífico de la energía atómica bajo el auspicio del programa Átomos para la Paz del presidente Eisenhower, pasando Irán (1958) a convertirse en miembro del Organismo Internacional de la Energía Atómica (OIEA). En el año 1968, Irán firmó el Tratado de No proliferación Nuclear. En 1972 firmó un acuerdo con Siemens para la construcción de la central nuclear en el puerto de Bushehren. (Cerio, 2016). En el año 1979, con la revolución Islámica y la caída del Sha, se interrumpen las investigaciones atómicas. Desde entonces, comenzaron los enfrentamientos entre Irán y los Estados Unidos, y las sanciones occidentales contra Irán a cuenta del desarrollo de su programa nuclear. En el contexto regional, es necesario señalar, que "el desarrollo nuclear para fines civiles ha constituido una reivindicación de muchos otros países de la zona, sin olvidar que tres de los cuatro Estados que no son parte del Tratado para la No Proliferación de Armamento Nuclear son vecinos de Irán y de los países árabes del Golfo, concretamente India, Pakistán $\mathrm{y}$, sobre todo, Israel, que se calcula almacena alrededor de doscientas cabezas nucleares" (Abu-warda Perez, Amal, 2000, p. 27).

En 1984, Estados Unidos cataloga a Irán como un Estado patrocinador del terrorismo. Con el presidente George W. Bush en la Casa Blanca, en 2002 declara a Irán, Irak, Corea del Norte como el "eje del mal". Funcionarios estadounidenses acusan a Teherán de operar un programa secreto de armas nucleares. Durante esta fase, se tensaron aún más las relaciones entre Irán y Occidente. Es a partir de 2002 cuando el programa nuclear iraní empezó a suponer una preocupación mayor para la comunidad internacional, cuando se descubrió que existían centros clandestinos de enriquecimiento de uranio. Desde entonces, la Unión Europea, que compartía las preocupaciones de la comunidad internacional en relación con el programa nuclear de Irán, apoyó y coordinó, a través de la entonces alta representante de la Unión para Asuntos Exteriores y Política de Seguridad y vicepresidenta de la Comisión, el Grupo de los Tres más Tres (E3+3: Francia, Alemania, Reino Unido, China, Rusia y EE. UU.) en sus largas negociaciones con Irán. El grupo de E3 de la Unión Europea consiguió que Irán aceptara suspender las actividades relacionada con el enriquecimiento de uranio, aunque por poco tiempo.

En octubre de 2003 los ministros de Asuntos Exteriores del EU-3 visitaron Irán para tratar el asunto nuclear. En noviembre de 2004 los cuatro países, con el apoyo del alto representante de la Unión Europea, Javier Solana, firmaban el "Acuerdo de París" cuyo objetivo era abrir un periodo de negociaciones que desembocaran en conseguir como resultado unas "garantías objetivas" de que el 
programa nuclear era exclusivamente para usos pacíficos. El Acuerdo de París fue la base de la resolución que la AIEA adoptó en su sesión de noviembre, donde los países europeos adoptaron una postura conciliadora. El Acuerdo de París es un acuerdo marco por el que las partes se concedían la oportunidad de negociar en términos definitivos el destino del programa nuclear iraní.

En posteriores negociaciones, los europeos, en lo relativo a la energía atómica, se comprometen a apoyar un programa nuclear civil seguro, económicamente viable y sin posibilidad de proliferación. Reconocen el derecho inalienable de Irán a tener energía nuclear y ofrecen a Teherán el combustible necesario, garantizando el aprovisionamiento permanente, pero exigen que Irán lo entregue una vez lo haya utilizado. A cambio, Teherán ha de comprometerse a no desarrollar actividades del ciclo de combustible más allá de las de construcción y de trabajo con reactores de agua ligera para investigación; a no enriquecer uranio; a no reprocesar el combustible; y a clausurar el reactor de agua pesada de Arak y las instalaciones de Natanz e Isfahán.(Lara Fernández, 2005, P.11). La propuesta de la Unión Europea fue rechazada por Irán con el argumento de que no le permitía realizar actividades de enriquecimiento. La Junta de Gobernadores del OIEA declaró que Irán incumplía sus obligaciones con arreglo al TNP. El 3 de enero de 2006 Irán anunció la reanudación de las operaciones de enriquecimiento de uranio. La reacción de EU-3 fue hacer pública una declaración (Berlín, 12 de enero) en la que denunciaba los incumplimientos de Irán y justificaba el envío del expediente al Consejo de Seguridad. El 31 de julio de 2006, intervino el Consejo de Seguridad aprobando una resolución en la que exigió a Irán la suspensión inmediata del proceso de enriquecimiento de uranio. Al año siguiente, el 24 de marzo, el Consejo de Seguridad adoptó la resolución 1747 con nuevas sanciones a Irán. Durante los años 2008 y 2009, la ONU adoptó nuevas resoluciones y sanciones contra Irán. El grupo E3/UE+3 emitió una declaración que reitera las propuestas de junio de 2006 -que también se adjuntaron a la resolución- y el compromiso respecto a la búsqueda de una solución negociada para alcanzar un acuerdo completo con Irán (Jonsson, 2007, p. 24).

Los años siguientes serán de desafío entre Irán y Occidente. Irán probó con éxito los misiles Seji capaces de alcanzar objetivos situados a 2.000 kilómetros. El año 2010, una nueva Resolución de Naciones Unidas, la 1929, declaraba que Irán no había demostrado que se hubiesen suspendido todas las actividades de enriquecimiento y reprocesamiento de uranio. En 2011, la OIEA denunció que Irán había adquirido la tecnología necesaria para fabricar una bomba nuclear, a lo que el Consejo de Seguridad de Naciones Unidas respondió elaborando otra batería de sanciones dirigidas esta vez contra las entidades financieras iraníes y que incluyó el embargo petrolero por parte de Estados Unidos y Unión Europea. 
Después de 12 años de negociaciones, en noviembre de 2013, tuvo lugar la firma de un preacuerdo entre el Grupo 5+1+UE (Alemania, China, Francia, Estados Unidos, Rusia y el Reino Unido) e Irán. En él, se contemplaba la suspensión temporal del programa nuclear iraní a cambio del levantamiento parcial de las sanciones y todo ello bajo la supervisión de la ONU. Este preacuerdo tendrá su continuidad hasta que se llegó a la firma definitiva del Acuerdo entre las partes, el 14 de julio de 2015. El 20 de julio de 2015, El Consejo de Seguridad de la ONU, aprobó por unanimidad una Resolución de apoyo al acuerdo nuclear alcanzado entre Irán y el grupo 5+1. El Acuerdo, conocido formalmente como «Plan de Acción Integral Conjunto», PAIC, constituye un hito histórico. El texto comienza señalando que "The E3/EU+3 (China, France, Germany, the Russian Federation, the United Kingdom and the United States, with the High Representative of the European Union for Foreign Affairs and Security Policy) and the Islamic Republic of Iran welcome this historic Joint Comprehensive Plan of Action (JCPOA), which will ensure that Iran's nuclear programme will be exclusively peaceful, and mark a fundamental shift in their approach to this issue. They anticipate that full implementation of this JCPOA will positively contribute to regional and international peace and security. Iran reaffirms that under no circumstances will Iran ever seek, develop or acquire any nuclear weapons." ('Joint Comprehensive Plan of Action Vienna, 14 July 2015', 2015). El PAIC, en su detallado contenido, establece el levantamiento total de las sanciones económicas y financieras que recaen sobre Irán, tanto las llevadas a cabo por el Consejo de Seguridad de Naciones Unidas y Unión Europea, como las que tienen carácter bilateral. Por su parte Irán, se compromete a cumplir unos límites estrictos y muy detallados al programa nuclear, cuyo cumplimiento se garantiza mediante un régimen de verificación mucho más estricto que el aplicado en los Acuerdos de Salvaguardias Generales por la OIEA. A pesar de sus limitaciones, el Acuerdo es positivo porque frena temporalmente la proliferación, introduce un régimen de verificación específico por la Agencia Internacional de la Energía Atómica, transforma un programa de enriquecimiento orientado al uso militar al uso pacífico, reduce las existencias de uranio enriquecido (de 8.000 a 300 kilos), reduce las centrifugadoras (a 5.060 durante 15 años) y limita el nivel de enriquecimiento del uranio al 3,7\% durante 15 años. En contrapartida, Irán logra el reconocimiento de su derecho a enriquecer el uranio en el futuro, lo que le permite convertirse en una potencia nuclear si así lo decide. Una capacidad que podrá desarrollar a medida que el Acuerdo prevé la modernización de sus centrifugadoras a partir de los 11 años. Además, el levantamiento de las sanciones de Naciones Unidas permitirá a Irán adquirir armas convencionales a partir de 2020 y desarrollar sus programas de misiles a partir de 2023, aunque persisten las sanciones bilaterales de EEUU 
y otros países sobre esas adquisiciones. ${ }^{7}$ El Acuerdo, solucionó alguno de los riesgos inmediatos de proliferación que generaba el programa nuclear y aplazó la solución de otros, incluidos el desarrollo de misiles balísticos y la injerencia en asuntos regionales para más adelante.

La negativa política del presidente de Estados Unidos, Donald Trump, al Acuerdo, llevó a los EE. UU. a retirarse unilateralmente de este en mayo de 2018 y restablecer plenamente sus sanciones en materia nuclear contra Irán en noviembre de 2018. En julio de 2019, como confirmó la OIEA, Irán comenzó a vulnerar las obligaciones derivadas del PAIC, y desde entonces ha ido incumpliendo gradualmente un número cada vez mayor de limitaciones. La Unión Europea, junto con los miembros restantes del grupo E3+3, mantiene su compromiso con la aplicación del PAIC. La jefa de la diplomacia europea, Federica Mogherini, lamentó la decisión y señaló que el bloque europeo está "determinado a preservar" el Acuerdo.

\section{La Unión Europea-Turquía, el proceso de adhesión}

Desde la promulgación de la república en Turquía, tras la Primera Guerra Mundial y la desintegración de Imperio Otomano, el acercamiento cultural con Europa ha ido acompañado de un incremento de las relaciones comerciales y políticas entre las dos partes. Por otro lado, el proceso de ampliación de la integración europea existe desde la creación de la CEE en 1957.

Turquía solicitó un estatus de asociado en 1959. El Consejo de Ministros de la CEE aceptó la solicitud y sugirió el establecimiento de una asociación hasta que las circunstancias de Turquía permitieran su adhesión. Las negociaciones subsiguientes dieron lugar a la firma del Acuerdo de asociación entre Turquía y la CEE, (el "Acuerdo de Ankara”) el 12 de septiembre de 1963. El Acuerdo de Ankara constituye la base jurídica de la asociación entre Turquía y la Unión Europea. El artículo 28 del Acuerdo establece que

Tan pronto como el funcionamiento del presente Acuerdo haya avanzado lo suficiente como para justificar la plena aceptación por parte de Turquía de las obligaciones derivadas del Tratado constitutivo de la Comunidad, las Partes contratantes examinarán la posibilidad de la adhesión de Turquía a la Comunidad ('Joint Comprehensive Plan of Action Vienna , 14 July 2015', 2015).

El Artículo establece que «el objetivo último de la relación entre Turquía y la CEE es la plena adhesión de Turquía a la Comunidad». En el Acuerdo

${ }^{7}$ La retirada unilateral de EEUU del Acuerdo nuclear con Irán: www.realinstitutoelcano.org > rielcano_es.

Araucaria. Revista Iberoamericana de Filosofia, Política, Humanidades y Relaciones Internacionales, año $22, \mathrm{n}^{\circ} 45$. Tercer cuatrimestre de 2020. Pp. 499-526. ISSN 1575-6823 e-ISSN 2340-2199 https://dx.doi.org/10.12795/araucaria.2020.i45.21 
de Ankara se preveían tres fases, la preparación, la transición y los períodos finales para la integración de Turquía en la CEE.

Con el Protocolo Adicional de 13 de noviembre de 1970, finalizó el período de preparación y se establecieron las condiciones para el período de transición. Se fijó un calendario de 22 años para el establecimiento de la Unión Aduanera. Sin embargo, en los años 70 las relaciones entre Turquía y la CEE fueron inestables políticamente y con el golpe militar del 12 de septiembre de 1980 se suspendieron las negociaciones.

Después del restablecimiento de la autoridad civil en Turquía en 1983, se inició un proceso de revitalización de las relaciones entre Turquía y la CEE. En 1987, Turquía solicitó la adhesión plena a la CEE, sin esperar a que se cumplimenten las fases previstas en el Acuerdo de Ankara. La negativa respuesta de la CEE a la solicitud de Turquía estaba motivada por las necesarias reformas que el Estado candidato debería realizar antes de la adhesión final. Tras dos años de negociaciones, la Unión Aduanera se estableció entre Turquía y la Unión Europea mediante la Decisión del Consejo de Asociación TurquíaCE de 6 de marzo de 1995. Así, las relaciones entre Turquía y la Asociación Unión Europea se llevaron al período final.

El Consejo Europeo de Helsinki, celebrado los días 10 y 11 de diciembre de 1999, produjo un gran avance en las relaciones entre Turquía y la Unión Europea. En Helsinki, Turquía fue reconocida oficialmente sin ninguna condición previa como Estado candidato en pie de igualdad con los demás Estados candidatos. El Consejo acogió «con satisfacción los positivos acontecimientos registrados recientemente en Turquía, señalados en el informe de situación de la Comisión, así como su propósito de proseguir las reformas con el fin de cumplir con los criterios de Copenhague. Turquía es un Estado candidato llamado a ingresar en la Unión atendiendo a los mismos criterios que se aplican a los demás Estados candidatos. Sobre la base de la estrategia europea actual, Turquía, al igual que otros Estados candidatos, podrá acogerse a una estrategia de preadhesión destinada a servir de estímulo y apoyo a sus reformas».(Durbán et al., 2006, P. 417). Como se preveía en las conclusiones del Consejo Europeo de Helsinki, la Comisión europea comenzó a preparar una Asociación para la Adhesión de Turquía, que fue aceptada el 8 de marzo de 2001.

Turquía prosiguió su proceso de reforma, que amplía el alcance de los derechos y libertades fundamentales, fortalece las reglamentaciones vigentes sobre democracia, Estado de derecho, libertad de pensamiento y expresión y derechos humanos. En este marco, entre 2002-2004 se aceptaron 8 paquetes de armonización, y entre 2001-20042 enmiendas constitucionales. 17 de diciembre de 2004, El Consejo Europeo ha acogido con satisfacción la adopción de los seis textos legislativos especificados por la Comisión. Ha decidido que, a la vista de lo anterior y del informe y recomendación de la Comisión, Turquía 
cumple suficientemente los criterios políticos de Copenhague para entablar las negociaciones de adhesión siempre y cuando aplique dichos textos legislativos. Ha invitado a la Comisión a que presente al Consejo una propuesta de marco de negociación con Turquía, basándose en el punto 23. Ha pedido al Consejo que apruebe dicho marco con miras a entablar las negociaciones el 3 de octubre de 2005. El Consejo ha adoptado la decisión de iniciar las negociaciones de adhesión con Turquía el 3 de octubre de 2005 (Durbán et al., 2004).

Como estaba previsto, las negociaciones de adhesión se iniciaron el 3 de octubre de 2005 y se anunció el Documento Marco de Negociación. Sin embargo, poco después, la relación entre Turquía y la Unión Europea entró en una nueva etapa llena de dificultades y debido a los bloqueos políticos de algunos Estados miembros y la cuestión de Chipre, el proceso de adhesión llegó a un punto muerto. La cuestión del reconocimiento de Chipre por parte de Turquía es un problema constante en todo el proceso negociador con la Unión Europea.

Desde 2005, las negociaciones para la adhesión plena de Turquía han progresado lentamente debido a que, de los 35 capítulos necesarios para completar el proceso de adhesión, solo 16 se habían abierto y uno se había cerrado en mayo de 2016. A principios de 2016, el acuerdo de refugiados entre Turquía y la Unión Europea tenía también por objetivo acelerar las negociaciones de adhesión, sin embargo, la Unión Europea ha criticado a Turquía por las violaciones de los derechos humanos y los déficits en el estado de derecho, suspendiendo las negociaciones. En 2017, los funcionarios de la Unión Europea expresaron que las políticas turcas violan los criterios de Copenhague para ser miembro de la Unión Europea. El 26 de junio de 2018, el Consejo de Asuntos Generales de la Unión Europea declaró que "The Council notes that Turkey has been moving further away from the European Union. Turkey's accession negotiations have therefore effectively come to a standstill and no further chapters can be considered for opening or closing and no further work towards the modernization of the EU-Turkey Customs Union is foreseen" (Council of the European Union, Brussels, 26 June 2018 (OR. en) 10555/18).

El 15 de marzo de 2019, el Consejo de Asociación Unión Europea-Turquía ha celebrado su 54. ${ }^{a}$ sesión en Bruselas. La sesión es la primera que se celebra después del levantamiento del estado de excepción el año pasado en Turquía, y ha brindado una ocasión útil para hacer balance de las relaciones Unión EuropeaTurquía y cambiar impresiones sobre una serie de asuntos. En la declaración final "La Unión Europea ha reiterado la importancia de las relaciones entre la Unión Europea y Turquía, país candidato y socio estratégico, y ha insistido en la importancia de un diálogo abierto y franco, dentro del marco establecido, con objeto de abordar los retos comunes en ámbitos de interés común como la migración, la lucha antiterrorista, la energía, el transporte, la economía y el 
comercio." El comunicado señala que "Turquía es para la Unión Europea un importante socio comercial, y la Unión Europea ha reiterado que los vínculos económicos entre Turquía y la Unión Europea son sólidos. Reconociendo los retos a los que se enfrenta la economía turca, la Unión Europea ha insistido en que es necesario abordar las vulnerabilidades conocidas para crear unas condiciones económicas y monetarias estables. Repasando las relaciones en el marco del Acuerdo de Asociación y de la Unión Aduanera, la Unión Europea ha instado a Turquía a que garantice que la actual Unión Aduanera funciona sin discriminaciones con respecto a todos los Estados miembros. (Council of the EU PRESS Declaración prensa Consejo de Asociación Unión EuropeaTurquía. Bruselas, 2019).

Después de décadas de negociación, reformas y los trabajos de modernización, entre muchas otras exigencias, hoy, las expectativas de adhesión de Turquía a la Unión Europea son parecidas a las que había al comienzo del proceso en los años sesenta. Analizando el pasado de las negociaciones, observando la actualidad de la Unión Europea, y pensando en el futuro del proceso de adhesión, que nadie sabe por cuánto tiempo se va a extender, no es difícil afirmar que la adhesión de Turquía a la Unión Europea no se dará ni a corto ni a medio plazos. Ambas partes deberían repensar sus relaciones y replantear los objetivos estratégicos de sus políticas exteriores. Las negociaciones Turquía-Unión Europea, no son exclusivamente bilaterales, más bien, son negociaciones multilaterales, Turquía-Unión Europea +27 . El proceso de adhesión de Turquía es excepcional por su realidad geopolítica y cultural. 


\section{Referencias bibliográficas:}

Ana María Bolborici and U.T., Brasov, "The european union and the Middle East peace process", en Bulletin of the Transilvania University of Brasov, Vol. 3 (52) (2010), pp. 9-14.

Amal Abu-warda Peréz, "La política exterior iraní. El Golfo Pérsico como área de influencia prioritaria”, en Estudios Internacionales de la Complutense, Volumen 12 / n 1 , (2010), pp. 9-30.

Ana Echagüe, "La Unión Europea y el Consejo de Cooperación para los Estados Árabes del Golfo Pérsico", en Working Paper / Documento de trabajo', Fride (2007).

Arab League, "State building for peace in the middle east: an EU action strategy" (2007).

Belen Lara Fernández, 'El programa nuclear de irán y la estabilidad estratégica en la zona' (2005).

Consejo de la Unión Europea, Bruselas, 24 de octubre de 2002, 13463/02, conclusiones de la presidencia (2002).

Consejo Europeo, D/99/1. Conclusiones de la Presidencia, - Consejo Europeo de Berlín, 24 y 25 de marzo de 1999, consejo europeo de berlín. Available at: https://ec.europa.eu > official > regulation > pdf > berlin_es

Council of the EU, "Declaración del alto representante, en nombre de la Unión Europea, sobre la situación en Libia”, PRESS ES, Comunicado de prensa 300/20 (2020).

Declaración a la prensa tras la sesión n ${ }^{\circ} 54$ del Consejo de Asociación UETurquía (Bruselas, 15 de marzo de 2019) (2019) Turkey 2019 Report, European Commission. Available at: https://ec.europa.eu/neighbourhoodenlargment/sites/near/files/20190529-turkey-report.pdf

European Council, 'Session of the European Council', Venice, 12 and 13 June 1980, (1980). Available at: papers2://publication/uuid/C9E49B8F-D1F748F7-8E6D-0075935B04AC

European Parlament, Consejo Europeo de Berlín, 24 y 25 de marzo de 1999. Declaración de la Presidencia. https:/www.europarl.europa.eu/summits/ ber2_es.htm.

Fernando Montoya Cerio, "Iran y su estrategico acuerdo nuclear", en Documentos de Opinión IEEE (Instituto Español de Estudios Estratégicos), (2016), pp. 1-14.

Joint Comprehensive Plan of Action Vienna, 14 July 2015, www.europarl. europa.eu > cmsdata, (2015).

Jonas Jonsson, “Irán y la UE: ¿Una relación estratégica?”, en Cuadernos de estrategia, $\mathrm{N}^{\mathrm{o}} 137,(2007)$, pp. 165-194. 
Joseph S. Roucek, "El Oriente Medio en la geopolítica”, en Revista de Política Internacional, 144, (1976), pp. 173-234.

Luis Pérez-Prat Durbán, “Turkuía y la Unión Europea: la larga marcha, ¿hacía la adhesión?», en Revista de Derecho Comunitario Europeo, núm. 24, Madrid, mayo-agosto (2006), pp. 401-437.

María Eleonora GuasconiI, "Europa Y La Primavera Árabe: Ambiciones Y Límites de la Política Mediterránea de la Unión Europea”, en ReDCE. Año 10, no 19 (2013), pp. 133-149.

María José Cervell Hortal, "La UE ante el lado más oscuro de la Primavera Árabe (Libia y Siria)”, en Boletín IEEE, 30 (2014), pp. 1-14. Available at: http://www.ieee.es.

Najib Abu-warda, "El conflicto palestino-israelí y la responsabilidad de la ONU" en Estudios Internacionales de la Complutense, Volumen 16 / n 2 (2012), pp. 29-54.

Najib Abu-Warda, "La Liga de los Estados Árabes ante los acontecimientos regionales" [en Najib Abu-Warda y Dolores Rubio García, coordinadores: La Primavera Árabe y sus perspectivas regionales e internacionales, Madrid, 2018], pp. 291-312.

Naxalli Calderón Hernández, "El proceso de Barcelona: Unión por el Mediterráneo y el Conflicto Árabe-Israelí. Elementos históricos”, en Europeans Review, (2016).

http://www.ieeiweb.eu/wp-content/uploads/2016/01/n4.pdf

Pilar Mellado, "Conferencia de paz para Oriente Medio en Madrid", en Revista de Derecho Político, No 38,(1994), pp. 303-359.

Rosa Martina Muñoz Fontela, "La Cooperación Política Europea en Oriente Medio", en Cuadernos de historia contemporánea, № 14 (1992), pp. 135 158.

Stelios Stavridis y Irene Fernández Molina, "El Parlamento Europeo y el conflicto de Libia ( 2011 ): ¿ una tribuna moral eficiente ?, en Revista CIDOB d'Afers Internacionals, n.101 ( 2013 ), pp. 153-176. 